\title{
APPROXIMATING COMMON FIXED POINTS OF FAMILIES OF QUASI-NONEXPANSIVE MAPPINGS
}

\author{
M. K. GHOSH \\ Department of Mathematics \\ Narajole Raj College \\ Midnapur, W B., INDIA \\ and

\section{LOKENATH DEBNATH} \\ Department of Mathematics \\ University of Central Florida \\ Orlando, Florida 32816, U S A.
}

(Received September 26,1993 and in revised form October 10, 1994)

\begin{abstract}
This paper deals with a family of quasi-nonexpansive mappings in a uniformly convex Banach space, and the convergence of iterates generated by this family. A fixed point theorem for two quasi-nonexpansive mappings is then proved. This theorem is then extended for a finite family of quasinonexpansive mappings. It is shown that Ishikawa's [1] result follows as special cases of results proved in this paper.
\end{abstract}

KEY WORDS AND PHRASES: Uniformly convex Banach spaces, fixed point theorems, families of quasi-nonexpansive mappings, and convergence of iterates.

1991 AMS SUBJECT CLASSIFICATION CODES: 47H10, 54H25.

\section{INTRODUCTION.}

Ishikawa [1] proved that if $T$ is a Lipschitzian pseudo-contractive map of a compact convex subset $E$ of a Hilbert space into itself and $x_{1}$ is any point in $E$, then the sequence

$$
x_{n+1}=\left(1-\alpha_{n}\right) x_{n}+\alpha_{n} T\left[\beta_{n} T x_{n}+\left(1-\beta_{n}\right) x_{n}\right]
$$

converges to a fixed point of $T$ where $\left\{\alpha_{n}\right\}$ and $\left\{\beta_{n}\right\}$ are sequences of positive numbers that satisfy certain conditions. De Marr [2] proved a theorem for existence of common fixed points for a commutative family of continuous (not necessarily linear) transformations. Diaz and Metcalf [3] considered the structure of the set of subsequential limit points of the sequence of iterates and investigated when this sequence converges. Dotson [4] applied Mann's iterative process to the approximation of fixed points of quasi-nonexpansive mappings in Hilbert space and in uniformly convex and strictly convex Banach spaces. He also generalized Mann's results to a locally convex Hausdorff linear topological space.

The main purpose of this paper is to consider a family of quasi-nonexpansive mappings and the convergence of iterates generated by this family. A fixed point theorem for two quasi-nonexpansive mappings is proved and is then extended for a finite family of mappings It is shown that results of this paper reduces to those of Ishikawa as special cases. 


\section{ITERATES GENERATED BY A FAMILY OF QUASI-NONEXPANSIVE MAPPINGS.}

Before we discuss our results, we state the following definition from Diaz and Metcalf [3] and a lemma due to Dotson [4]

DEFINITION. Suppose $B$ is a Banach space and $D$ is a convex subset of $B$ A mapping $T$ is said to be quasi-nonexpansive on a subset $D$ of $B$ provided $T$ maps $D$ into itself, and if $p \in D$ and $T p=p$ then $\|T x-p\| \leq\|x-p\|$ hold for all $x \in D$.

LEMMA 2.1 If $\left\{v_{n}\right\}$ and $\left\{w_{n}\right\}$ are sequences in the closed unit ball of a uniformly convex Banach space and if $\left\{z_{n}\right\}=\left\{\left(1-\alpha_{n}\right) v_{n}+\alpha_{n} w_{n}\right\}$ satisfies $\lim _{n \rightarrow \infty}\left\|z_{n}\right\|=1$ where $0<a \leq \alpha_{n} \leq b<1$, then

$$
\lim _{n \rightarrow \infty}\left\|v_{n}-w_{n}\right\|=0 \text {. }
$$

Suppose $D$ is a convex subset of a Banach space $B$, and $T$ and $S$ are two self mappings of $D$. For a $x_{1} \in D$, we define a sequence $\left\{x_{n}\right\}_{n=1}^{\infty}$ such that

$$
x_{n+1}=\left(1-\alpha_{n}\right) S x_{n}+\alpha_{n} T y_{n},
$$

where

$$
y_{n}=\left(1-\beta_{n}\right) S x_{n}+\beta_{n} T x_{n},
$$

$\alpha_{n}$ and $\beta_{n}$ satisfy (i) $0<a \leq \alpha_{n} \leq b<1$, and (ii) $0 \leq \beta_{n} \leq \beta<1$. If $S=I$, the identity mapping, the iterates (2.2) are identical with those of Ishikawa

We now state the following condition:

Condition $C$ : The mappings $T, S: D \rightarrow D$ are said to satisfy condition $C$ if there exists a nondecreasing function $f:[0, \infty)$ with $f(0)=0$ and $f(r)>0$ for $r \in(0, \infty)$ such that

$$
\|T y-S x\| \geq f(d(x, \mathrm{~F}))
$$

for all $x, y \in D$ with $y=(1-\mu) S x+\mu T x$, where $0 \leq \mu \leq \beta<1$ and $F$ is the common fixed point set of $T$ and $S$.

If we set $S=I$ in (2.4), then condition $C$ becomes identical with condition A as stated by Maiti and Ghosh [5].

We now state our main result for two quasi-nonexpansive mappings:

THEOREM 2.1 Suppose $D$ is a closed convex subset of a uniformly convex Banach space $B$ and $T, S$ are two quasi-nonexpansive mappings of $D$ into itself. If $T, S$ satisfy condition $C$, then, for any $x_{1} \in D$, sequence (2.2) converges to a member of the common fixed point set $F$ of $T$ and $S$.

PROOF. If $x_{1} \in F$, then the result follows trivially. We assume $x_{1} \in D-F$. Then, for an arbitrary $p \in F$, we have, from (2.2),

$$
\begin{aligned}
\left\|x_{n+1}-p\right\| & =\left\|\left(1-\alpha_{n}\right) S x_{n}+\alpha_{n} T y_{n}-p\right\| \\
& =\left\|\left(1-\alpha_{n}\right)\left(S x_{n}-p\right)+\alpha_{n}\left(T y_{n}-p\right)\right\| \\
& \leq\left(1-\alpha_{n}\right)\left\|S x_{n}-p\right\|+\alpha_{n}\left\|T y_{n}-p\right\| \\
& \leq\left(1-\alpha_{n}\right)\left\|x_{n}-p\right\|+\alpha_{n}\left\|y_{n}-p\right\| \\
& =\left(1-\alpha_{n}\right)\left\|x_{n}-p\right\|+\alpha_{n}\left\|\left(1-\beta_{n}\right) S x_{n}+\beta_{n} T x_{n}-p\right\| \\
& =\left(1-\alpha_{n}\right)\left\|x_{n}-p\right\|+\alpha_{n}\left\|\left(1-\beta_{n}\right)\left(S x_{n}-p\right)+\beta_{n}\left(T x_{n}-p\right)\right\| \\
& \leq\left(1-\alpha_{n}\right)\left\|x_{n}-p\right\|+\alpha_{n}\left(1-\beta_{n}\right)\left\|S x_{n}-p\right\|+\alpha_{n} \beta_{n}\left\|T x_{n}-p\right\| \\
& \leq\left(1-\alpha_{n}\right)\left\|x_{n}-p\right\|+\alpha_{n}\left(1-\beta_{n}\right)\left\|x_{n}-p\right\|+\alpha_{n} \beta_{n}\left\|x_{n}-p\right\| \\
& =\left\|x_{n}-p\right\| .
\end{aligned}
$$

This implies that $d\left(x_{n+1}, F\right) \leq d\left(x_{n}, F\right)$ and hence that the sequence $\left\{d\left(x_{n}, F\right)\right\}$ is nonincreasing. Thus $\lim _{n \rightarrow \infty} d\left(x_{n}, F\right)$ exists. We next show that this limit is zero. 
Suppose, if possible, $\lim _{n \rightarrow \infty} d\left(x_{n}, F\right)=l>0$. Then, for $p \in F, \lim _{n \rightarrow \infty}\left\|x_{n}-p\right\| l^{\prime} \geq l>0$ We choose a positive integer $N$ such that $\left\|x_{n}-p\right\| \leq 2 l^{\prime}$ for $n \geq N$. Now, we set

$$
v_{n}=\frac{S x_{n}-p}{\left\|x_{n}-p\right\|}, w_{n} \frac{T y_{n}-p}{\left\|x_{n}-p\right\|} .
$$

Then $\left\|v_{n}\right\| \leq 1,\left\|w_{n}\right\| \leq 1$ for all $n$. This implies that the sequences $\left\{v_{n}\right\}$ and $\left\{w_{n}\right\}$ are in the closed unit ball of $B$. Further,

$$
\begin{aligned}
\lim _{n \rightarrow \infty}\left\|\left(1-\alpha_{n}\right) v_{n}+\alpha_{n} w_{n}\right\| & =\lim _{n \rightarrow \infty} \frac{\left\|\left(1-\alpha_{n}\right)\left(S x_{n}-p\right)+\alpha_{n}\left(T y_{n}-p\right)\right\|}{\left\|x_{n}-p\right\|} \\
& =\lim _{n \rightarrow \infty} \frac{\left\|\left(1-\alpha_{n}\right) S x_{n}+\alpha_{n} T y_{n}-p\right\|}{\left\|x_{n}-p\right\|} \\
& =\lim _{n \rightarrow \infty} \frac{\left\|x_{n+1}-p\right\|}{\left\|x_{n}-p\right\|}=\frac{l^{\prime}}{l^{\prime}}=1 .
\end{aligned}
$$

Thus the sequences $\left\{v_{n}\right\},\left\{w_{n}\right\}$ and $\left\{\left(1-\alpha_{n}\right) v_{n}+\alpha_{n} w_{n}\right\}$ satisfy all the conditions of Lemma 2.1 and hence we must have

$$
\lim _{n \rightarrow \infty}\left\|v_{n}-w_{n}\right\|=0
$$

But for $n \geq N$, we have

$$
\left\|v_{n}-w_{n}\right\|=\frac{\left\|T y_{n}-S x_{n}\right\|}{\left\|x_{n}-p\right\|} \geq \frac{f\left(d\left(x_{n}, F\right)\right)}{\left\|x_{n}-p\right\|} \geq \frac{f(l)}{2 l^{\prime}}>0 .
$$

Thus we conclude that $\lim _{n \rightarrow \infty}\left\|v_{n}-w_{n}\right\| \neq 0$, which is a contradiction. Thus $\lim _{n \rightarrow \infty} d\left(x_{n}, F\right)=0$. We now show that this implies $\left\{x_{n}\right\}$ converges to a member of $F$. Since $\lim _{n \rightarrow \infty} d\left(x_{n}, F\right)=0$. For a given $\epsilon>0$ there exists $N_{\epsilon}>0$ and $y_{\epsilon} \in F$ such that $\left\|x_{N_{\epsilon}}-y_{\epsilon}\right\|<\epsilon$, which implies $\left\|x_{n}-y_{\epsilon}\right\|<\epsilon$ for all $n \geq N_{\epsilon}$. Thus, if $\epsilon_{k}=2^{-k}$ for $k \in P$, where $P$ is the set of positive integers, then corresponding to each $\epsilon_{k}$, there is an $N_{k}>0$ and a $y_{k} \in F$ such that $\left\|x_{n}-y_{k}\right\| \leq \frac{\epsilon_{k}}{4}$ for all $n \geq N_{k}$. We require $N_{k+1} \geq N_{k}$ for all $k \in P$. We have, for all $k \in P$,

$$
\left\|y_{k}-y_{k+1}\right\|=\left\|y_{k}-x_{N_{k+1}}+x_{N_{k+1}}-y_{k+1}\right\|<\frac{\epsilon_{k}}{4}+\frac{\epsilon_{k+1}}{4}=\frac{3 \epsilon_{k+1}}{4}
$$

We now suppose that $S(y, \epsilon)=\{x \in B:\|x-y\| \leq \epsilon\}$ represents the closed sphere with center at $y$ of radius $\epsilon$. For $x \in S\left(y_{k+1}, \epsilon_{k+1}\right)$, we have

$$
\left\|y_{k}-x\right\|=\left\|y_{k}-y_{k+1}+y_{k+1}-x\right\|<\left(\frac{3 \epsilon_{k+1}}{4}+\epsilon_{k+1}\right)<2 \epsilon_{k+1}=\epsilon_{k} .
$$

Thus

$$
S\left(y_{k+1}, \epsilon_{k+1}\right) \subseteq S\left(y_{k}, \epsilon_{k}\right) \quad \text { for } k \in P .
$$

Thus, $\left\{S\left(y_{k}, \epsilon_{k}\right)\right\}$ is a nested sequence of nonvoid closed spheres with radii $\epsilon_{k}$ tending to zero. By the Cantor intersection theorem, $\bigcap_{k \in P} S\left(y_{k}, \epsilon_{k}\right)$ contains exactly one point $p$. Since $T$ and $S$ are quasinonexpansive, the fixed point set $F$ is closed by a result due to Dotson [6], and the sequence $\left\{y_{k}\right\}$ from $F$ converges to $p$, and hence $p \in F$. In view of the result

$$
\left\|x_{n}-y_{k}\right\|<\frac{1}{4} \epsilon_{k} \quad \text { for } n \geq N_{k}
$$

the sequence $\left\{x_{n}\right\}_{n=1}^{\infty}$ converges to $p$. This completes the proof. 


\section{AN EXTENSION OF THEOREM 2.1.}

We extend Theorem 2.1 for the case of more than two mappings. For the sake of clarity, we consider three quasi-nonexpansive mappings $T, S, R$ of $D$ into itself. For any $x_{1} \in \mathrm{D}$, we define a sequence $\left\{x_{n}\right\}_{n=1}^{\infty}$ such that

$$
x_{n+1}=\alpha_{n}^{1} R x_{n}+\alpha_{n}^{2} S x_{n}+\alpha_{n}^{3} T y_{n}
$$

with

$$
y_{n}=\beta_{n}^{1} R x_{n}+\beta_{n}^{2} S x_{n}+\beta_{n}^{3} T x_{n}
$$

where,

$$
\begin{gathered}
0<a \leq \alpha_{n}^{2} \leq b<1, \quad \sum_{i=1}^{3} \alpha_{n}^{2}=1 \\
0 \leq \beta_{n}^{i} \leq \beta<1, \quad \sum_{i=1}^{3} \beta_{n}^{2}=1
\end{gathered}
$$

In the present situation, it is necessary to modify Condition A as stated by Maiti and Ghosh [5] in the following way.

Condition D. The mappings $T, S, R: D \rightarrow D$ are said to satisfy condition $D$, if there exists a nondecreasing function $f:[0, \infty) \rightarrow[0, \infty)$ with $f(0)=0$ and $f(r)>0$ for $r \in(0, \infty)$ such that

$$
\|T y-\lambda S x-(1-\lambda) R x\| \geq f(d(x, F)), \lambda \in(0,1)
$$

for all $x, y \in D$ with $y_{1}=a_{1} R x+a_{2} S x+a_{3} T x$, where $0 \leq a_{\imath} \leq \beta<1$ with $\sum_{i=1}^{3} a_{i}=1$ and $F$ is the common fixed point set of $T, S, R$ in $D$.

If we set $S=R=I$ in (3.5), this condition reduces to condition $A$ as stated in a paper by Maiti and Ghosh [5].

THEOREM 3.1 Suppose $D$ is a closed convex subset of a uniformly convex Banach space $B$. If $T$, $S, R$ are three quasi-nonexpansive and self-mappings of $D$ satisfying condition $D$, then, for any $x_{1} \in D$, the sequence (3.1) with (3.3) and (3.4) converges to an element of the common fixed point set $F$.

PROOF. The proof of this theorem is similar to that of Theorem 2.1. It suffices to show that

$$
\lim _{n \rightarrow \infty} d\left(x_{n}, F\right)=0
$$

If possible, we let $\lim _{n \rightarrow \infty} d\left(x_{n}, F\right)=l>0$. Then for $p \in F, \lim _{n \rightarrow \infty}\left\|x_{n}-p\right\|=l^{\prime} \geq l>0$. We now choose a positive integer $N$ such that $\left\|x_{n}-p\right\| \leq 2 l^{\prime}$ for $n \geq N$.

We now write

$$
v_{n}=\frac{\alpha_{n}^{1}}{1-\alpha_{n}^{3}} \cdot \frac{R x_{n}-p}{\left\|x_{n}-p\right\|}+\frac{\alpha_{n}^{2}}{1-\alpha_{n}^{3}} \cdot \frac{S x_{n}-p}{\left\|x_{n}-p\right\|}
$$

and

$$
w_{n}=\frac{T y_{n}-p}{\left\|x_{n}-p\right\|}
$$

Then 


$$
\begin{aligned}
\left\|v_{n}\right\| & \leq \frac{\alpha_{n}^{1}}{1-\alpha_{n}^{3}}\left(\frac{\left\|R x_{n}-p\right\|}{\left\|x_{n}-p\right\|}\right)+\frac{\alpha_{n}^{2}}{1-\alpha_{n}^{3}}\left(\frac{\left\|S x_{n}-p\right\|}{\left\|x_{n}-p\right\|}\right) \\
& \leq \frac{\alpha_{n}^{1}+\alpha_{n}^{2}}{1-\alpha_{n}^{3}}=1
\end{aligned}
$$

and

$$
\left\|w_{n}\right\| \leq \frac{\left\|T y_{n}-p\right\|}{\left\|x_{n}-p\right\|} \leq \frac{\left\|x_{n}-p\right\|}{\left\|x_{n}-p\right\|}=1 .
$$

Thus the sequence $\left\{v_{n}\right\}$ and $\left\{w_{n}\right\}$ are in the closed unit ball of $B$ Now,

$$
\begin{aligned}
\lim _{n \rightarrow \infty}\left\|\left(1-\alpha_{n}^{3}\right) v_{n}+\alpha_{n}^{3} w_{n}\right\| & =\lim _{n \rightarrow \infty} \frac{\left\|\alpha_{n}^{1}\left(R x_{n}-p\right)+\alpha_{n}^{2}\left(S x_{n}-p\right)+\alpha_{n}^{3}\left(T y_{n}-p\right)\right\|}{\left\|x_{n}-p\right\|} \\
& =\lim _{n \rightarrow \infty} \frac{\left\|\alpha_{n}^{1} R x_{n}+\alpha_{n}^{2} S x_{n}+\alpha_{n}^{3} T y_{n}-p\right\|}{\left\|x_{n}-p\right\|} \\
& =\lim _{n \rightarrow \infty} \frac{\left\|x_{n+1}-p\right\|}{\left\|x_{n}-p\right\|}=\frac{l^{\prime}}{l^{\prime}}=1 .
\end{aligned}
$$

Then, by Lemma 2.1, we must have $\lim _{n \rightarrow \infty}\left\|w_{n}-v_{n}\right\|=0$ But, for $n \geq N$, we get

$$
\begin{aligned}
\left\|w_{n}-v_{n}\right\| & =\frac{1}{\left\|x_{n}-p\right\|}\left\|\left(T y_{n}-p\right)-\frac{\alpha_{n}^{1}}{1-\alpha_{n}^{3}}\left(R x_{n}-p\right)-\frac{\alpha_{n}^{2}}{1-\alpha_{n}^{3}}\left(S x_{n}-p\right)\right\| \\
& =\frac{1}{\left\|x_{n}-p\right\|}\left\|T y_{n}-\frac{\alpha_{n}^{1}}{1-\alpha_{n}^{3}} R x_{n}-\frac{\alpha_{n}^{2}}{1-\alpha_{n}^{3}} S x_{n}\right\| \\
& \geq \frac{f\left(d\left(x_{n}, F\right)\right)}{\left\|x_{n}-p\right\|} \geq \frac{f(l)}{2 l^{\prime}}>0 .
\end{aligned}
$$

This implies that $\lim _{n \rightarrow \infty}\left\|w_{n}-v_{n}\right\| \neq 0$ which leads to a contradiction, and hence $\lim _{n \rightarrow \infty} d\left(x_{n}, F\right)=0$ This completes the proof.

It is obvious that the above results may be extended readily to any finite family of quasinonexpansive mappings.

We now give a variant of Theorem 21.

THEOREM 3.2 Suppose $D$ is a closed, bounded and convex subset of a uniformly convex Banach space $B$. If $T$ and $S$ are two commuting and non-expansive self-mappings of $D$ satisfying condition $\mathrm{C}$, then, for any $x_{1} \in D$, sequence (2.2) converges to a member of the common fixed point set $F$ of $T$ and $S$.

PROOF. In Theorem 2.1, the existence of common fixed point set $F$ is assumed, whereas the existence of $F$ is ensured here by a Theorem of Bowder [7] Since, with the existence of fixed points, nonexpansive mappings are also quasi-nonexpansive, the present theorem may be treated as a special case of Theorem 21.

Similarly, a variant of Theorem 31 may be obtained.

REMARK. If, in Theorem $32, D$ is assumed to be compact and convex (which is a stronger condition), then the existence of common fixed point set $F$ is guaranteed by the theorem of DeMarr [2]

ACKNOWLEDGMIENT. The authors express grateful thanks to Professor M Maiti for his help and interest in the work This work was partially supported by the University of Central Florida 


\section{REFERENCES}

[1] ISHIKAWA, S., "Fixed points by a new iteration method," Proc. Amer. Math. Soc. 44 (1974), 147. 150.

[2] DeMARR, R., "Common fixed points for commuting contraction mappings," Pacific J. Math. 13 (1963), 1139-1141.

[3] DIAZ, J.B. and METCALF, F.T., "On the structure of the set of subsequential limit points of successive approximations," Bull. Amer. Math. Soc. 73 (1967), 516-519.

[4] DOTSON, W.G. Jr., "On the Mann iterative process," Trans. Amer. Math. Soc. 149 (1970), 65-73.

[5] MAITI, M. and GHOSH, M.K., "Approximating fixed points by Ishikawa iterates, Bull. Austral. Math. Soc. 40 (1989), 113-117.

[6] DOTSON, W.G., "Fixed points of quasi-nonexpansive mappings," J. Austral. Math. Soc. 13 (1972), 167-170.

[7] BROWDER, F.E., "Nonexpansive nonlinear operators in a Banach space," Proc. Nat. Acad. Sci. U.S.A. 54 (1965), 1041-1044. 


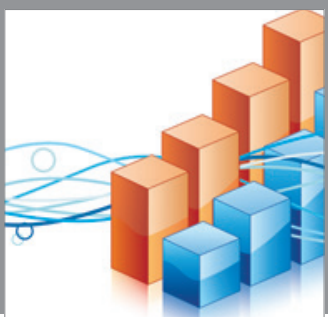

Advances in

Operations Research

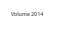

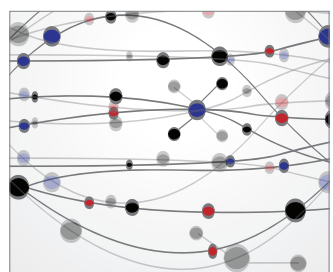

\section{The Scientific} World Journal
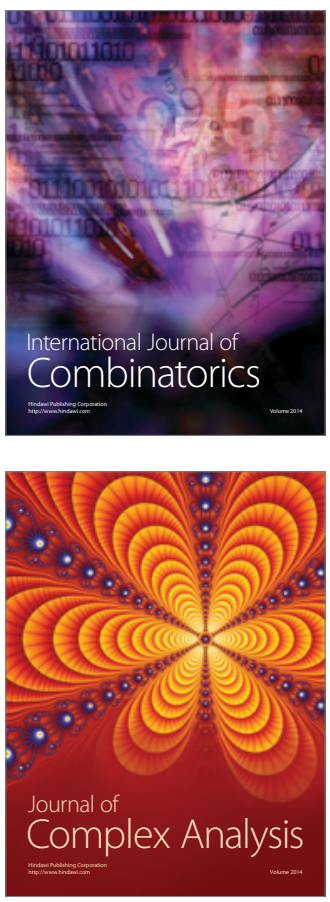

International Journal of

Mathematics and

Mathematical

Sciences
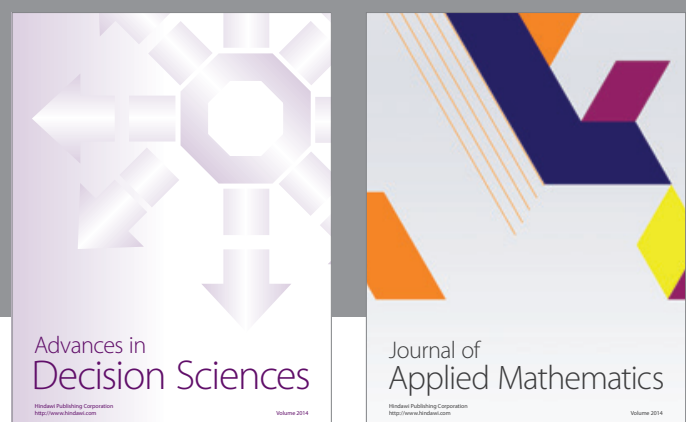

Journal of

Applied Mathematics
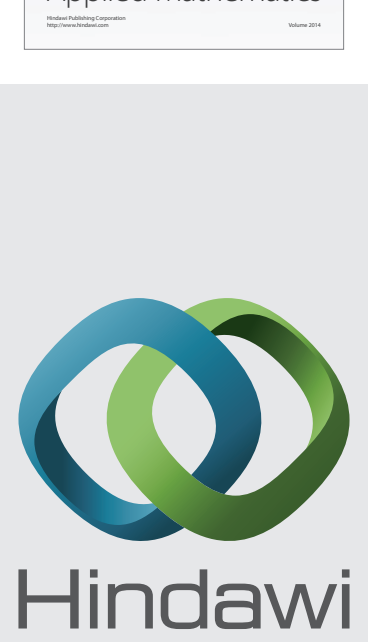

Submit your manuscripts at http://www.hindawi.com
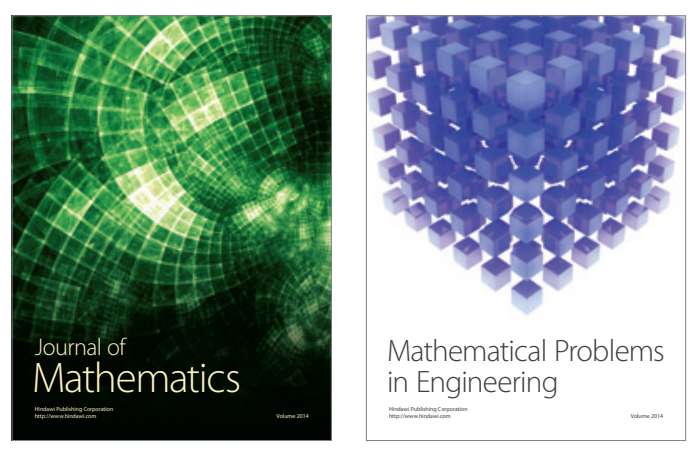

Mathematical Problems in Engineering
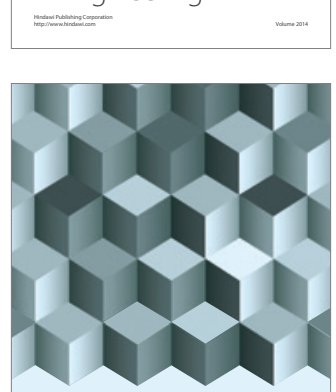

Journal of

Function Spaces
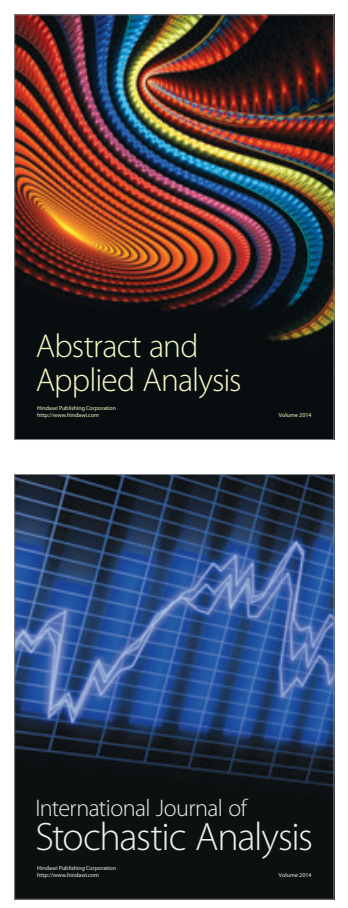

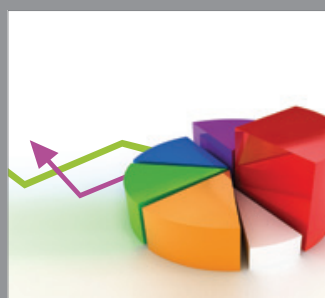

ournal of

Probability and Statistics

Promensencen
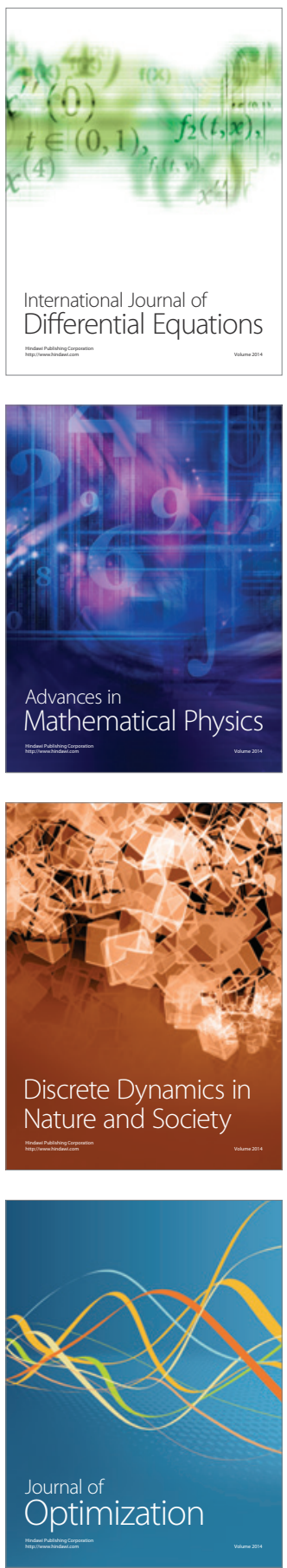